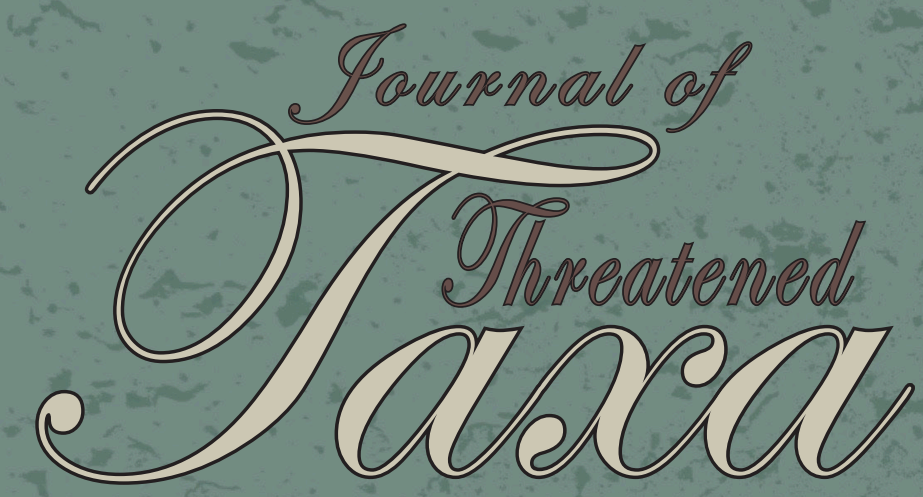

Building evidence for conservation globally
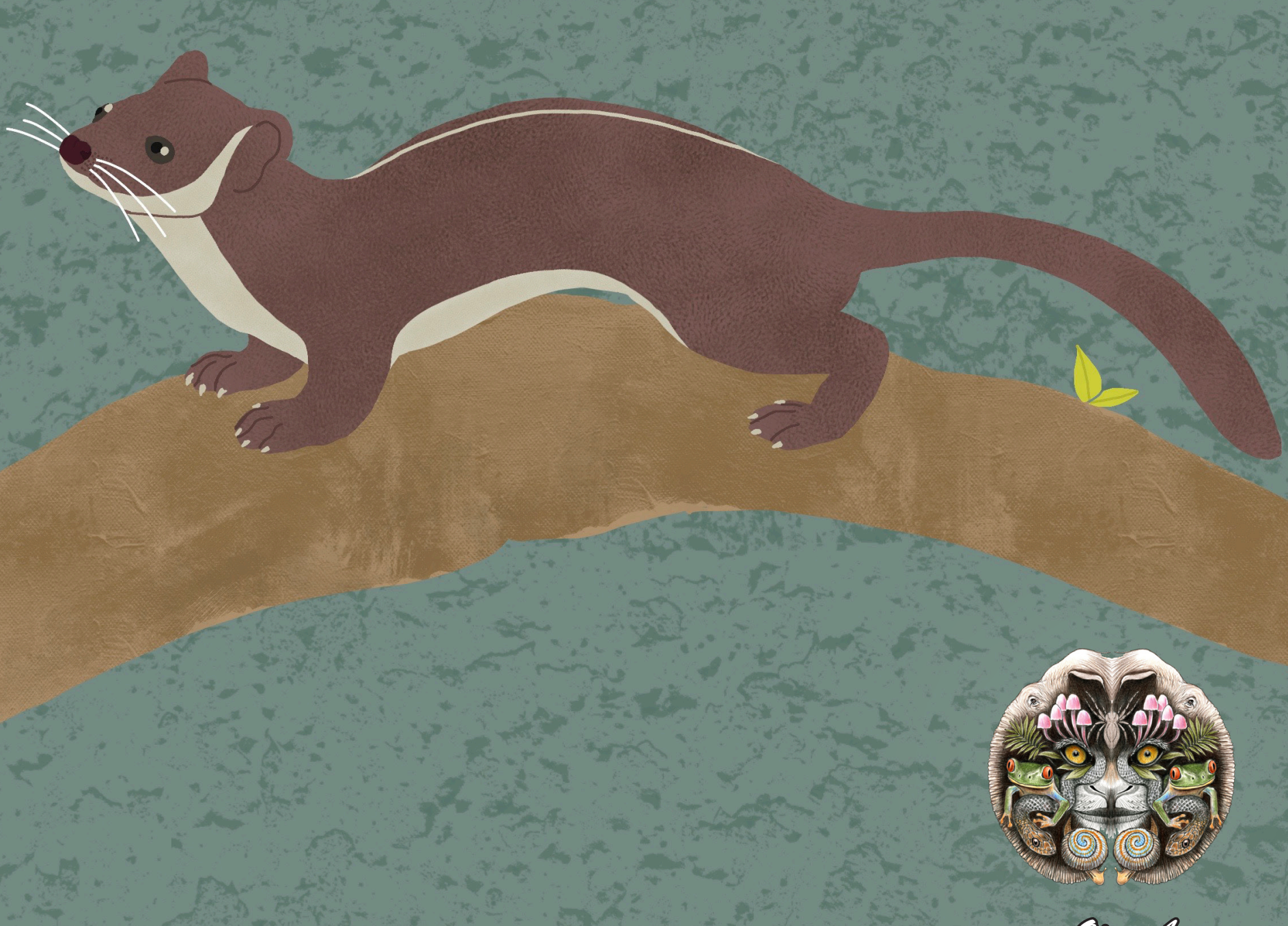

Qpecosecess

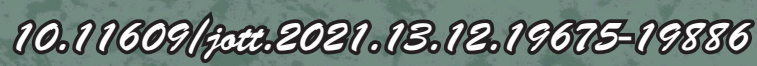
cocosurthreatecredtassararg

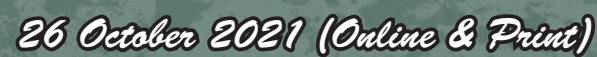

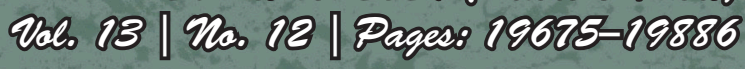




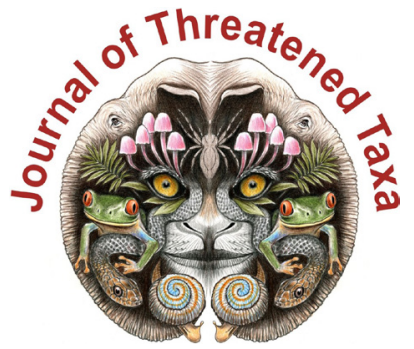

ISSN 0974-7907 (Online); ISSN $0974-7893$ (Print)

Publisher

Host

Wildlife Information Liaison Development Society

www.wild.zooreach.org

Zoo Outreach Organization www.zooreach.org

No. 12, Thiruvannamalai Nagar, Saravanampatti - Kalapatti Road, Saravanampatti, Coimbatore, Tamil Nadu 641035, India

Ph: +91 9385339863 | www.threatenedtaxa.org

Email: sanjay@threatenedtaxa.org

EDITORS

\section{Founder \& Chief Editor}

Dr. Sanjay Molur

Wildlife Information Liaison Development (WILD) Society \& Zoo Outreach Organization (ZOO)

12 Thiruvannamalai Nagar, Saravanampatti, Coimbatore, Tamil Nadu 641035, India

\section{Deputy Chief Edito}

Dr. Neelesh Dahanukar

Noida, Uttar Pradesh, India

\section{Managing Editor}

Mr. B. Ravichandran, WILD/ZOO, Coimbatore, India

\section{Associate Editors}

Dr. Mandar Paingankar, Government Science College Gadchiroli, Maharashtra 442605, India Dr. Ulrike Streicher, Wildlife Veterinarian, Eugene, Oregon, USA

Ms. Priyanka Iyer, ZOO/WILD, Coimbatore, Tamil Nadu 641035, India

Dr. B. A. Daniel, $200 /$ WIID, Coimbatore, Tamil Nadu 641035, India

\section{Editorial Board}

Dr. Russel Mittermeie

Executive Vice Chair, Conservation International, Arlington, Virginia 22202, USA

\section{Prof. Mewa Singh Ph.D., FASc, FNA, FNASc, FNAPsy}

Ramanna Fellow and Life-Long Distinguished Professor, Biopsychology Laboratory, and Institute of Excellence, University of Mysore, Mysuru, Karnataka 570006, India; Honorary Professor, Jawaharlal Nehru Centre for Advanced Scientific Research, Bangalore; and Adjunct Professor, National Institute of Advanced Studies, Bangalore

\section{Stephen D. Nash}

Scientific Illustrator, Conservation International, Dept. of Anatomical Sciences, Health Sciences Center, T-8, Room 045, Stony Brook University, Stony Brook, NY 11794-8081, USA

\section{Dr. Fred Pluthero}

Toronto, Canada

\section{Dr. Priya Davidar}

Sigur Nature Trust, Chadapatti, Mavinhalla PO, Nilgiris, Tamil Nadu 643223, India

\section{Dr. Martin Fisher}

Senior Associate Professor, Battcock Centre for Experimental Astrophysics, Cavendish

Laboratory, JJ Thomson Avenue, Cambridge CB3 OHE, UK

\section{Dr. John Fellowes}

Honorary Assistant Professor, The Kadoorie Institute, 8/F, T.T. Tsui Building, The University of Hong Kong, Pokfulam Road, Hong Kong

\section{Prof. Dr. Mirco Solé}

Universidade Estadual de Santa Cruz, Departamento de Ciências Biológicas, Vice-coordenado do Programa de Pós-Graduação em Zoologia, Rodovia Ilhéus/Itabuna, Km 16 (45662-000)

Salobrinho, Ilhéus - Bahia - Brasil

\section{Dr. Rajeev Raghavan}

Professor of Taxonomy, Kerala University of Fisheries \& Ocean Studies, Kochi, Kerala, India

\section{English Editors}

Mrs. Mira Bhojwani, Pune, India

Dr. Fred Pluthero, Toronto, Canad

Mr. P. Ilangovan, Chennai, India

Web Maintenance

Mrs. Latha G. Ravikumar, ZOO/WILD, Coimbatore, India

\section{Typesetting}

Mr. Arul Jagadish, ZOO, Coimbatore, India

Mrs. Radhika, ZOO, Coimbatore, India

Mrs. Geetha, ZOO, Coimbatore India

\section{Fundraising/Communications}

Mrs. Payal B. Molur, Coimbatore, India

Subject Editors 2018-2020

Fungi

Dr. B. Shivaraju, Bengaluru, Karnataka, India

Dr. R.K. Verma, Tropical Forest Research Institute, Jabalpur, India

Dr. Vatsavaya S. Raju, Kakatiay University, Warangal, Andhra Pradesh, India

Dr. M. Krishnappa, Jnana Sahyadri, Kuvempu University, Shimoga, Karnataka, India

Dr. K.R. Sridhar, Mangalore University, Mangalagangotri, Mangalore, Karnataka, India

Dr. Gunjan Biswas, Vidyasagar University, Midnapore, West Bengal, India

Plants

Dr. G.P. Sinha, Botanical Survey of India, Allahabad, India

Dr. N.P. Balakrishnan, Ret. Joint Director, BSI, Coimbatore, India

Dr. Shonil Bhagwat, Open University and University of Oxford, UK

Prof. D.J. Bhat, Retd. Professor, Goa University, Goa, India

Dr. Ferdinando Boero, Università del Salento, Lecce, Italy

Dr. Dale R. Calder, Royal Ontaro Museum, Toronto, Ontario, Canada

Dr. Cleofas Cervancia, Univ. of Philippines Los Baños College Laguna, Philippines

Dr. F.B. Vincent Florens, University of Mauritius, Mauritius

Dr. Merlin Franco, Curtin University, Malaysia

Dr. V. Irudayaraj, St. Xavier's College, Palayamkottai, Tamil Nadu, India

Dr. B.S. Kholia, Botanical Survey of India, Gangtok, Sikkim, India

Dr. Pankaj Kumar, Kadoorie Farm and Botanic Garden Corporation, Hong Kong S.A.R., China

Dr. V. Sampath Kumar, Botanical Survey of India, Howrah, West Bengal, India

Dr. A.J. Solomon Raju, Andhra University, Visakhapatnam, India

Dr. Vijayasankar Raman, University of Mississippi, USA

Dr. B. Ravi Prasad Rao, Sri Krishnadevaraya University, Anantpur, India

Dr. K. Ravikumar, FRLHT, Bengaluru, Karnataka, India

Dr. Aparna Watve, Pune, Maharashtra, India

Dr. Qiang Liu, Xishuangbanna Tropical Botanical Garden, Yunnan, China

Dr. Noor Azhar Mohamed Shazili, Universiti Malaysia Terengganu, Kuala Terengganu, Malaysia Dr. M.K. Vasudeva Rao, Shiv Ranjani Housing Society, Pune, Maharashtra, India

Prof. A.J. Solomon Raju, Andhra University, Visakhapatnam, India

Dr. Mandar Datar, Agharkar Research Institute, Pune, Maharashtra, India

Dr. M.K. Janarthanam, Goa University, Goa, India

Dr. K. Karthigeyan, Botanical Survey of India, India

Dr. Errol Vela, University of Montpellier, Montpellier, France

Dr. P. Lakshminarasimhan, Botanical Survey of India, Howrah, India

Dr. Larry R. Noblick, Montgomery Botanical Center, Miami, USA

Dr. K. Haridasan, Pallavur, Palakkad District, Kerala, India

Dr. Analinda Manila-Fajard, University of the Philippines Los Banos, Laguna, Philippines

Dr. P.A. Sinu, Central University of Kerala, Kasaragod, Kerala, India

Dr. Afroz Alam, Banasthali Vidyapith (accredited A grade by NAAC), Rajasthan, India

Dr. K.P. Rajesh, Zamorin's Guruvayurappan College, GA College PO, Kozhikode, Kerala, India

Dr. David E. Boufford, Harvard University Herbaria, Cambridge, MA 02138-2020, USA

Dr. Ritesh Kumar Choudhary, Agharkar Research Institute, Pune, Maharashtra, India

Dr. Navendu Page, Wildlife Institute of India, Chandrabani, Dehradun, Uttarakhand, India

Invertebrates

Dr. R.K. Avasthi, Rohtak University, Haryana, India

Dr. D.B. Bastawade, Maharashtra, India

Dr. Partha Pratim Bhattacharjee, Tripura University, Suryamaninagar, India

Dr. Kailash Chandra, Zoological Survey of India, Jabalpur, Madhya Pradesh, India

Dr. Ansie Dippenaar-Schoeman, University of Pretoria, Queenswood, South Africa

Dr. Rory Dow, National Museum of natural History Naturalis, The Netherlands

Dr. Brian Fisher, California Academy of Sciences, USA

Dr. Richard Gallon, llandudno, North Wales, LL30 1UP

Dr. Hemant V. Ghate, Modern College, Pune, India

Dr. M. Monwar Hossain, Jahangirnagar University, Dhaka, Bangladesh

Mr. Jatishwor Singh Irungbam, Biology Centre CAS, Branišovská, Czech Republic.

Dr. lan J. Kitching, Natural History Museum, Cromwell Road, UK

Dr. George Mathew, Kerala Forest Research Institute, Peechi, India

Dr. John Noyes, Natural History Museum, London, UK

For Focus, Scope, Aims, and Policies, visit https://threatenedtaxa.org/index.php/JoTT/aims_scope
For Article Submission Guidelines, visit https://threatenedtaxa.org/index.php/JoTT/about/submissions
For Policies against Scientific Misconduct, visit https://threatenedtaxa.org/index.php/JoTT/policies_various

continued on the back inside cover 


\title{
On the pteridophytes of Bherjan-Borajan-Padumoni Wildlife Sanctuary, Assam, India
}

\author{
Pranjal Borah ${ }^{1}$ (D) \& Jayanta Barukial ${ }^{2}$ (D)
}

\begin{abstract}
${ }^{1}$ Department of Life Sciences, Dibrugarh University, Dibrugarh, Assam 786004, India.
2 P.G. Department of Life Sciences, Debraj Roy College, Golaghat, Assam 785621, India.

${ }^{1}$ borahpranjal27@gmail.com (corresponding author), ${ }^{2}$ barukialjayanta@gmail.com
\end{abstract}

Abstract: A preliminary survey on the pteridophytic flora of Bherjan-Borajan-Padumoni Wildlife Sanctuary of Assam, India revealed a total of 33 species belonging to 23 genera and 15 families. Most of the species belong to the family Pteridaceae followed by Polypodiaceae and Thelypteridaceae. A brief taxonomic description of each species is provided. Stenochlaena palustris (Burm.f.) Bedd., an edible fern, grows abundantly all over, especially in the openings and clearings.

Keywords: Fern, Polypodiaceae, Pteridophytic flora, Pteridaceae, Stenochlaena, taxonomic description, Thelypteridaceae.

Citation: Borah, P. \& J. Barukial (2021). On the pteridophytes of Bherjan-Borajan-Padumoni Wildlife Sanctuary, Assam, India. Journal of Threatened Taxa 13(12): 19781-19790. https://doi.org/10.11609/jott.7488.13.12.19781-19790

Copyright: (C) Borah \& Barukial 2021. Creative Commons Attribution 4.0 International License. JoTT allows unrestricted use, reproduction, and distribution of this article in any medium by providing adequate credit to the author(s) and the source of publication.

Funding: Self-funded.

Competing interests: The authors declare no competing interests.

Author details: PRANJAL BORAH is a PhD scholar at Dibrugarh University, Assam, pursuing research in the area of plant taxonomy. DR. JAYANTA BARUKIAL is the Associate Professor and Head of the Department of Botany and PG Department of Life Sciences, Debraj Roy College, Assam and a recognized PhD supervisor of Dibrugarh University. His research interest is in the field of plant taxonomy, cytology, molecular taxonomy, environmental issues.

Author contributions: All authors equally contributed to the present study.

Acknowledgements: The authors are grateful to the principal chief conservator of forest (wildlife), Assam and the Assam State Biodiversity Board for giving the necessary permissions for field surveys. Also thankful to local forest officials for providing necessary help and guidance during the field trips. The authors are also grateful to Dr. Pankaj Chetia, asst. professor, Dept. of Life Sciences, Dibrugarh University, Assam and Mr. Nakul Neog, asst. professor, Sadiya College, Assam for their advice and help. 


\section{INTRODUCTION}

Pteridophytes, also known as ferns and fern-allies, flowerless and seedless plants that once dominated the world vegetation 280-230 million years ago. The main plant body is sporophytic and the dominant phase in its life cycle. Chapman (2009) estimates that there are about 12,000 species of ferns and fern-allies across the world. According to Moran (2015), "worldwide, there are about 13,600 species of ferns and lycophytes". According to a survey, the pteridophytic flora of India comprises around 1,000 species belonging to 191 genera and 67 families including 47 endemic Indian ferns (Dixit 1984) and in another report, more than 1,100 species of pteridophytes belonging to 144 genera and 34 families with about 235 endemic species (Chandra 2000) from India. According to Fraser-Jenkins et al. (2017), "altogether there are about 1135 species including 42 exotics and 53 further subspecies", from the Indian subcontinent.

The Bherjan-Borajan-Padumoni is one of the smallest wildlife sanctuaries of Assam covering $7.22 \mathrm{~km}^{2}$ of area spreading across three blocks located in Tinsukia district of the Upper Brahmaputra Valley of Assam, India which consists of three separate forests, namely Bherjan, Borajan, and Padumoni. The forest is the home for an endangered primate species, i.e., Western Hoolock Gibbon. Bherjan, Borajan, and Padumoni are tiny isolated pockets of lowland tropical forest covering an area of 105 ha, 493 ha, and 176 ha respectively and an ideal habitat for primate species like the Bengal Slow Loris, Assamese Macaque, Pig-tailed Macaque, Rhesus Macaque, Capped Langur, Stump-tailed Macaque, and Western Hoolock Gibbon. The three areas are disjoint and unconnected by tea gardens and human settlement. These are entirely on the flat plains of the Brahmaputra Valley. There are small, scattered marshes, with swamps, covered with dense growth of grass and Alpinia herb. The natural vegetation of all these areas is tropical wet evergreen 'rainforest' type. Bherjan is almost entirely covered with trees with a closed canopy. The original vegetation has been replaced by a fairly old mixed plantation dominated by the deciduous species Lagerstroemia parviflora and Terminalia myriocarpa. The Padumoni part is mostly in a degraded state due to large-scale felling. The canopy has been very badly broken up. Only a few mature trees of Artocarpus, Bombax, Lagerstroemia, and Mesua species can be seen. Borajan is a pocket of excellent rainforest, dominated by Dipterocarpus macrocarpus. Except for Teak, all the species as in the other two forest pockets are found. Bamboo species are found in all areas.
In Assam, a few systematic works on pteridophytes have been accomplished, like Kachroo (1953), Panigrahi (1960, 1968), Panigrahi \& Chowdhury $(1961,1962)$, Dutta et al. (1980), Handigue \& Konger (1986), Kachroo et al. (1989), Borthakur et al. (2001, 2018), Devi \& Majumdar (2003), Sen \& Ghosh (2011), and Kalita (2015).

\section{METHODS}

A number of field visits were undertaken to the study area in the year 2019-2020 (Figure 1). The different pteridophytes were collected from a range of habitat; however, those species which are Red Listed (IUCN) or used as food or shelter were left untouched. Under such circumstances, digital photographs of the concerned species were taken. The specimens were preserved and studied with standard literature like Baishya \& Rao (1982), Jamir \& Rao (1988), and Borthakur et al. $(2001,2018)$. The plants were collected from the field, cleaned, and pressed for the preparation of herbarium. Herbariums are prepared by following standard herbarium technique (Jain \& Rao 1977) and deposited at the Botany Department, Debraj Roy College, Golaghat, Assam. Digital photographs of the specimens were also taken and some of them are displayed (Image 1).

The families are enumerated in text according to Fraser-Jenkins (2009). However, the genera and species within the families are listed alphabetically. The taxonomic citation is based on published literature and IPNI, Tropicos, and The Plant List.

\section{RESULTS}

A total of 33 species of pteridophytes belonging to 23 genera and 15 families are recorded. The plants are enumerated with a brief taxonomic description of each species.

\section{Selaginellaceae}

1. Selaginella monospora Spring; Collection No. DRC- 5005.

Terrestrial. Stem about $40 \mathrm{~cm}$ long, prostrate, ascending, branched. Leaves dimorphic, green, midrib prominent, lateral leaves ovate. Strobilus up to $8 \mathrm{~mm}$ long, terminal, simple, sporophylls spiral, spore green.

2. Selaginella semicordata (Wall. ex Hook. \& Grev.) Spring; Collection No. DRC-5006.

Terrestrial. Stem slender, branched with related 

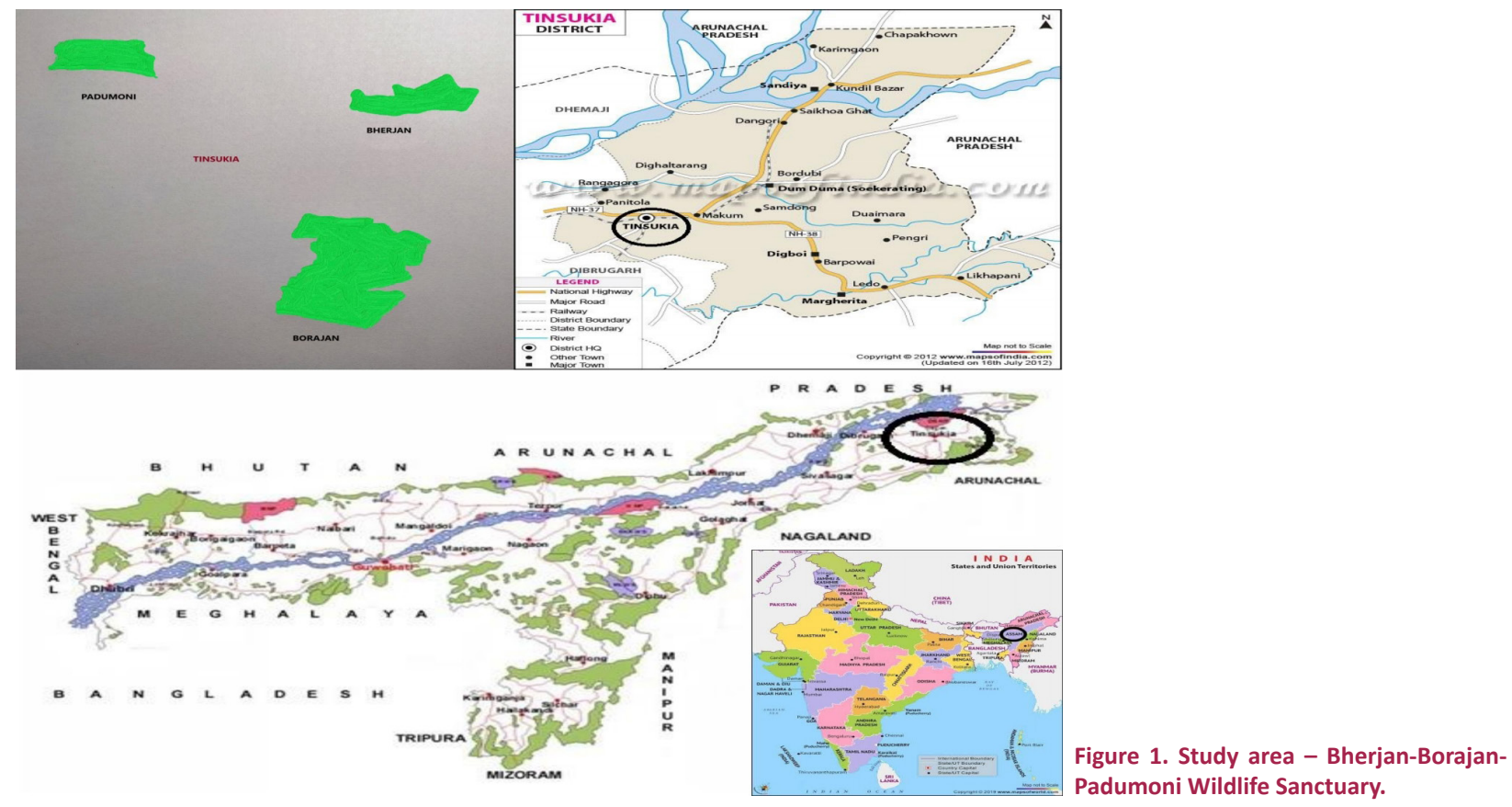

dichotomy, rhizophore arises from forking. Leaves spirally arranged, lanceolate, entire, membranous. Strobilus up to $0.6 \mathrm{~cm}$ long, sporophyll lanceolate, ciliate at base; sporangia oval, orange in colour, spore oval, dark brown.

\section{Marattiaceae}

3. Angiopteris evecta (G.Forst.) Hoffm.; Collection No. DRC- 5015.

Terrestrial. Tree like fern. Rhizome erect, cylindrical, broad. Stipes swollen at base, adaxially flattened, abaxially rounded, whitish linear streaks all over, with small brown scales and minute hairs. Lamina bipinnate; pinnae subopposite, with long swollen stalk, oblonglanceolate, veins simple or forked twice, almost parallel, reaching the margin. Sori sub-marginal, ellipsoid; sporangia up to six pairs in two rows, which is boatshaped. Spores hyaline, tetrahedral, pale green.

\section{Gleicheniaceae}

4. Dicranopteris linearis (Burm.f.) Underw.; Collection No. DRC- 5016.

Terrestrial. Rhizome long creeping, densely covered by hairs, scales absent; hairs minute, multicellular, reddish-brown. Stipes slender, straggling, rigid and polished; apical bud covered by brown hairs. Fronds pinnate or dichotomously branched; margin curved, deeply covered with brown hairs; rachises repeatedly forked, covered with dark-brown hairs; veins prominent, 2-forked, free; lamina light green; texture hard. Sori small, globose, without paraphyses, in the two rows on both sides of the costa. Spores numerous, trilete, deeply grooved.

\section{Polypodiaceae}

5. Microsorum pteropus

Copel.; Collection No. DRC- 5021. Aquatic. Rhizome creeping, fleshy, green, apex scaly; lanceolate. Fronds simple or $3 / 5$ - lobate, lanceolate, veins distinct above and below, main veins rather wide apart, lamina very dark, dirty green, often black when dry. Sori small, globose, scattered irregularly within the main areoles; sporangia slender stalked, oval. Spores yellowish-green.

6. Microsorum punctatum (L.) Copel.; Collection No. DRC- 5025.

Epiphyte. Rhizome short creeping. Fronds without distinct stipe, simple, sessile, lanceolate or elliptic, base decurrent, margin entire, midrib across at the base, veins visible but not prominent; pinnae glabrous above and below, dark green when fresh, blackish when dry. Sori numerous, small, round, irregularly scattered on upper half of the frond; sporangia oval, short-stalked. Spores yellowish-green.

7. Pyrrosia Ianceolata (L.) Farewell.; Collection No. DRC- 5029.

Epiphyte. Rhizome wide creeping, slender, clothed with scales; base rounded, margin profusely hairy, entire. Lamina simple, lanceolate acute apex, base decurrent, entire or wavy, green and glossy above, brownish below, 
upper surface glabrous, lower surface densely covered by stellate hairs, veins immersed; lamina wrinkle up on drying. Sori irregularly distributed on the anterior half of lamina; sporangia dark-brown, naked. Spores greenishyellow.

8. Pyrrosia piloselloides (L.) M.G.Price; Collection No.:- DRC-5032.

Epiphyte. Rhizome long creeping, clothed with scales; lamina dimorphic, simple; sterile lamina sessile or shortly stalked, roundish or obovate, base cuneate, margin entire; stipe of fertile frond scaly at base, grooved adaxially, straw-coloured; fertile lamina linear to oblong, apex round, base decurrent, margin entire; veins indistinct. Sori marginal, linear, continuous along the tip of lamina; sporangia oval, short stalked darkbrown. Spores oval to elliptic, light-brown.

\section{Lygodiaceae}

9. Lygodium flexuosum (L.) Sw.; Collection No. DRC5037.

Climber. Rhizome creeping, short, covered by darkbrown, multicellular, uniseriate hairs. Stipes glabrous, abaxially rounded, adaxiaily flattened, dark-brown. Fronds widespreading, tripinnate, glabrous; primary pinnae alternate, bears two to three pinnules alternately; pinnules oblong-lanceolate, simple or terminal leaflets forked, basal leaflets often large, separate or lobed with 2-3 leaflets, sterile leaflets finely toothed; texture firm; rachis and costa densely or sparsely pubescent all over; veins distinct, free, reaching the margin; fertile leaflets a little narrower than the sterile ones. Sporangia arranged adaxially protruding from the margin; sporangia large, short stalked, about five pairs, arranged in two rows, alternate.

10. Lygodium japonicum (Thunb.) Sw.; Collection No. DRC- 5038.

Climber. Rhizome long creeping, covered with black hairs. Stipes scaly at base. Fronds wide-spreading, tripinnate; primary pinnae forked, opposite; fertile pinnae much contracted giving an appearance of dissected lamina; texture herbaceous; primary rachis scabrous; secondary rachis ridged; veins distinct, free, reaching the margin. Sori finger like, marginal projections which are greenish when young and dark brown at maturity; sporangia short stalked, arranged in two rows, alternate. Spores small, numerous.

\section{Pteridaceae}

11. Acrostichum heterophyllum L.; Collection No. DRC- 5040.

Epiphytes. Rhizome long creeping, densely covered by scales; apex acuminate, centre dark brown, palebrown in the rest. Stipes of sterile fronds covered with scales similar to rhizome. Lamina dimorphous, simple or elliptic, apex rounded, margin entire; costa and veins indistinct, immersed; texture thick, fleshy, more or less covered by stellate hairs when young, sparsely or rarely when matured, lamina pale or dark-green; linear oblong, apex rounded. Sori confluent along the tip of lamina, sporangia oval, short stalked, intermingled with stellate hairs, dark-brown. Spores oval to elliptic, hyaline, lightbrown.

12. Adiantum capillus-veneris L.; Collection No. DRC5055.

Terrestrial. Rhizome short creeping, densely clothed with brown, narrow, lanceolate, acuminate, entire scales; Stipes slender scaly at base, glabrous and dark glossy above. Lamina bipinnate, lanceolate, cuneate at base, lower margin straight or concave and entire, outer margin rounded, lobed, lobes crenate, sterile lobes with more or less rounded, finely toothed edges; rachis uncovered; veins dichotomously branched. Sori elliptic or linear; sporangia globose, small and short stalked. Spores smooth walled.

13. Pityrogramma calomelanos (L.) Link.; Collection No. DRC- 5057.

Terrestrial. Rhizome short, erect, glossy above, black. Lamina oblong-triangular, subcoriaceous, bipinnate, pinnae in the lower half of the lamina more or less equal; gradually shortened towards apex, lower surface covered with white waxy powdery substance, lobes oblique, elliptic, toothed; rachis black-ebeneous, glossy; veins dichotomously radiated, free. Sori continuous throughout the lower surface. Spores tetrahedral.

14. Pteris biaurita L.; Collection No. DRC- 5045.

Terrestrial. Rhizome erect, short, scaly at the apex; linear-lanceolate, margin hairy, dark brown. Stipes glabrous except at base, adaxially grooved. Lamina bipinnatifid, glabrous; pinnae up to 12 pairs, subopposite, lanceolate, sessile or shortly stalked, margin lobed, apex rounded, margin entire; pinnae pale green; veins distinct, other veins forked once. Sori confluent along the margin of the sinus but not reaching the apex of the lobes. Spores tetrahedral, dark brown.

15. Pteris ensiformis Burm.f.; Collection No. DRC5046.

Terrestrial. Rhizome erect, short, scaly; scales linear-lanceolate, entire, shining, dark-brown. Stipes glabrous, polished, slender, abaxially rounded, adaxially grooved. Fronds dimorphous, but sometimes some fronds partly fertile and partly sterile; Fertile lamina bi-pinnate, glabrous; the segments very narrow and 

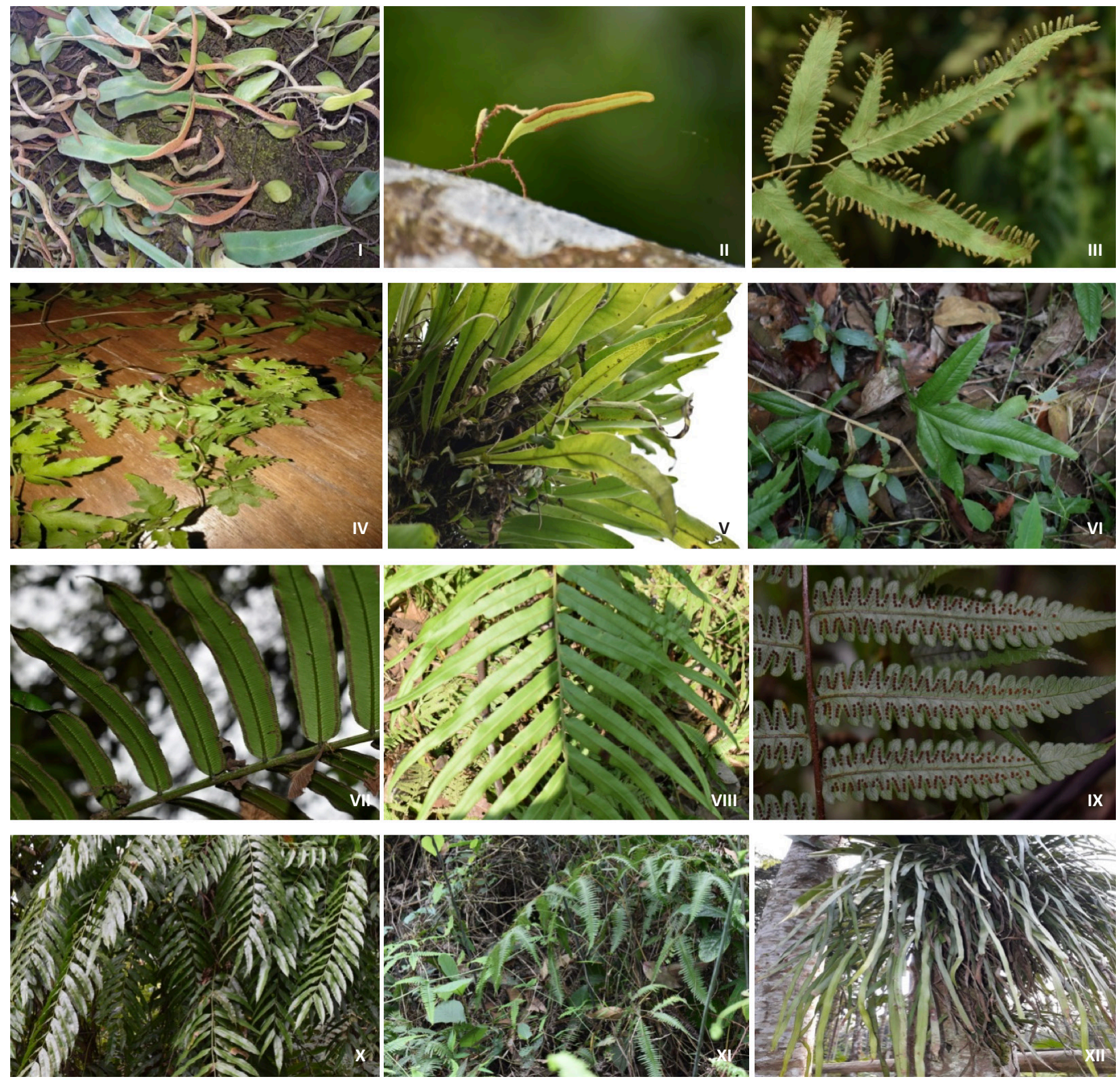

Image 1. I-Pyrrosia lanceolata (L.) Farw. | II-Acrostichum heterophyllum L. | III-Lygodium flexuosum (L.) Sw. | IV-Lygodium japonicum (Thunb.) Sw. | V-Microsorum punctatum (L.) Copel. | VI-Microsorum pteropus (BI.) Copel. | VII-Angiopteris evecta (G.Forst.) Hoffm. | VIIIBlechnum orientale L. | IX-Cyathea gigantea (Wall. ex Hook.) Holtt. | X-Stenochlaena palustris (Burm.) Bedd. | XI-Dicranopteris linearis (Burm.f.) Underw. | XII-Vittaria elongata Sw. @ Pranjal Borah

elongated, entire; lamina pale-green; rachis glabrous, grooved above, pale-brown; veins distinct on both surfaces, simple or forked, free. Sori confluent, marginal, developing basipetally. Spores dark brown, tetrahedral.

16. Pteris longipinnula Wall. ex J. Agardh.; Collection No. DRC- 5047.

Terrestrial. Rhizome erect, densely clothed with scales; scales linear, reddish-brown. Stipes erect, slender, abaxially rounded, adaxially grooved, scaly at base. Lamina bipinnatifid, with 3 - 8 pairs of lateral pinnae and an apical pinnae, apex caudate; lobes gradually reduced towards apex, often auricled; rachis and surface of the pinnae uncovered; spinules present on upper surface; veins free, forked once. Sori marginal, continuous nearly to the apex of the lobes. Spores triangular to ' $T$ '-shaped, dark brown.

17. Pteris semipinnata L.; Collection No. DRC- 5048.

Terrestrial. Rhizome short-creeping, densely scaly; dark brown. Stipes erect, tufted, slender, glabrous, dark brown at base, pale brown above. Lamina pinnate, ovate-lanceolate, glabrous; upper part of lamina cut down nearly to the rachis into numerous close entire 
linear lobes; costae and costules minutely grooved on the upper surface; veins free, fine, forked once. Sori linear, continuous along the margins of lobes except quite near reaching the sinus. Spores triangular to oval, pale brown.

18. Pteris vittata L.; Collection No. DRC- 5049.

Terrestrial. Rhizome suberect, short, densely covered by scales at apex, membranaceous, entire, pale-brown. Stipes pale-brown, clothed with linear, silky, pale-brown scales. Lamina simple pinnate with a single, elongate, linear, terminal pinnae like the lateral ones; pinnae numerous, opposite or subopposite, reduced to deflexed auricles, all pinnae sessile, linear-lanceolate, acuminate at apex; veins simple or forked once, free, distinct on both surfaces. Sori all along the margin, except at base and apex; indusia membranaceous. Spores round, yellowish-green.

19. Vittaria elongata Sw.; Collection No. DRC- 5053.

Epiphyte. Rhizome short creeping, thick, slender branched, densely scaly. Stipes flattened. Lamina simple, linear-oblong lanceolate, gradually tapering towards both ends, apex acuminate, margin entire, midrib distinct; veins slightly distinct above and below, simple, immersed, parallel; lamina dark green. Sori linear, confluent; sporangia short stalk. Spore pale yellowishgreen.

\section{Cyatheaceae}

20. Cyathea gigantea (Wall. ex Hook.) Holtt.

Terrestrial. Tree like fern. Trunk massive, erect, densely covered by scales dark brown. Stipes tufted, glossy, scaly at base, glabrous above, abaxially rounded, adaxially grooved, dark purple. Lamina bipinnate, deltoid, dark- green when fresh, brownish when dry; oblong-lanceolate, alternate, shortly stalked, about 20 $\mathrm{cm}$ apart, acuminate at apex about $2 \mathrm{~cm}$ apart, apex acuminate, margin lobed half way to the costae, rounded apex, margin crenate; texture of lamina herbaceous; rachis slightly dark brown. Sori median on the veins, spherical and inverted ' $V$ ' shape, exindusiate; sporangia numerous, compact.

21. Cyathea spinulosa Wall. ex Hook.

Terrestrial. Tree like fern. Trunk erect, up to $3 \mathrm{~m}$ or more tall. Stipes and rachis strongly aculeate or spiny, scales linear-lanceolate, long hair-pointed, black. Lamina bipinnate, short stalked; rachis of pinnules and main veins of lobes scaly below, but the latter glabrous above; lamina coriaceous; veins usually forked once, free. Sori large, round, indusium completely covering the sorus when young, soon breaking irregularly.

\section{Dennstaedtiaceae}

22. Microlepia speluncae (L.) Moore; Collection No. DRC- 5063.

Terrestrial. Rhizome long creeping, stout, hairy; palebrown. Stipes rounded below, grooved above, short hairy, purplish-green. Lamina tripinnate or quadripinnate; pinnae about eight pairs, alternate, petiolate; ovatelanceolate, apex acuminate, largest pinnule narrowly deltoid, subopposite or alternate, shortly stalked, basal acroscopic leaflet much larger than the rest; ultimate pinnules, veins slightly distinct, forked once, free, not reaching the margin. Sori submarginal, near the base of the sinuses between the lobes, indusia cup-shaped, hairy; sporangia copious. Spores yellowish-brown.

\section{Lindsaeaceae}

23. Sphenomeris chinensis (L.) Maxon; Collection No. DRC- 5067.

Terrestrial. Rhizome short creeping, stout, covered by scales; scales hair-like, stiff, dark-brown. Lamina tripinnate or quadripinnatifid, distal part bipinnatifid, lanceolate, apex acuminate, acroscopic base truncate, basiscopic base cuneate; secondary pinnae about eight pairs, alternate, shortly stalked, acute; tertiary pinnae about three pairs, alternate, shortly stalked, obovate, apex rounded, base cuneate. Sori marginal or submarginal at the end of veins; indusial attached basally. Spores bilateral and brown.

\section{Thelypteridaceae}

24. Amblovenatum opulentum (Kaulf.) J.P.Roux.; Collection No. DRC- 5069.

Terrestrial. Rhizome creeping, densely scaly; scales narrow, linear brown. Stipes erect, slender, grooved, hairy at grooves, scaly at base. Lamina simple pinnate, lanceolate, apex acuminate; pinnae about 20-30 pairs, alternate or subopposite, sessile; apex obtuse or rounded, margin wavy, clothed with small yellowish glandular hairs; lamina dark green. Sori mostly confined to lobes, globose, often much immersed and visible as punch form dots on the upper surface; indusia thin, sporangia slender stalked. Spores dark in colour.

25. Christella parasitica (L.) H.Lev.; Collection No. DRC- 5072.

Terrestrial. Rhizome creeping, densely scaly; linearlanceolate, apex acuminate, margin more or less clothed with short, soft hairs. Simple pinnate, pinnae numerous, alternate or subopposite, sessile, margin lobed twothird to the costa, lobes up to 20 pairs, oblique, basal acroscopic lobe slightly larger than the others; rachis copiously covered by long and short hairs; costa, eostules 

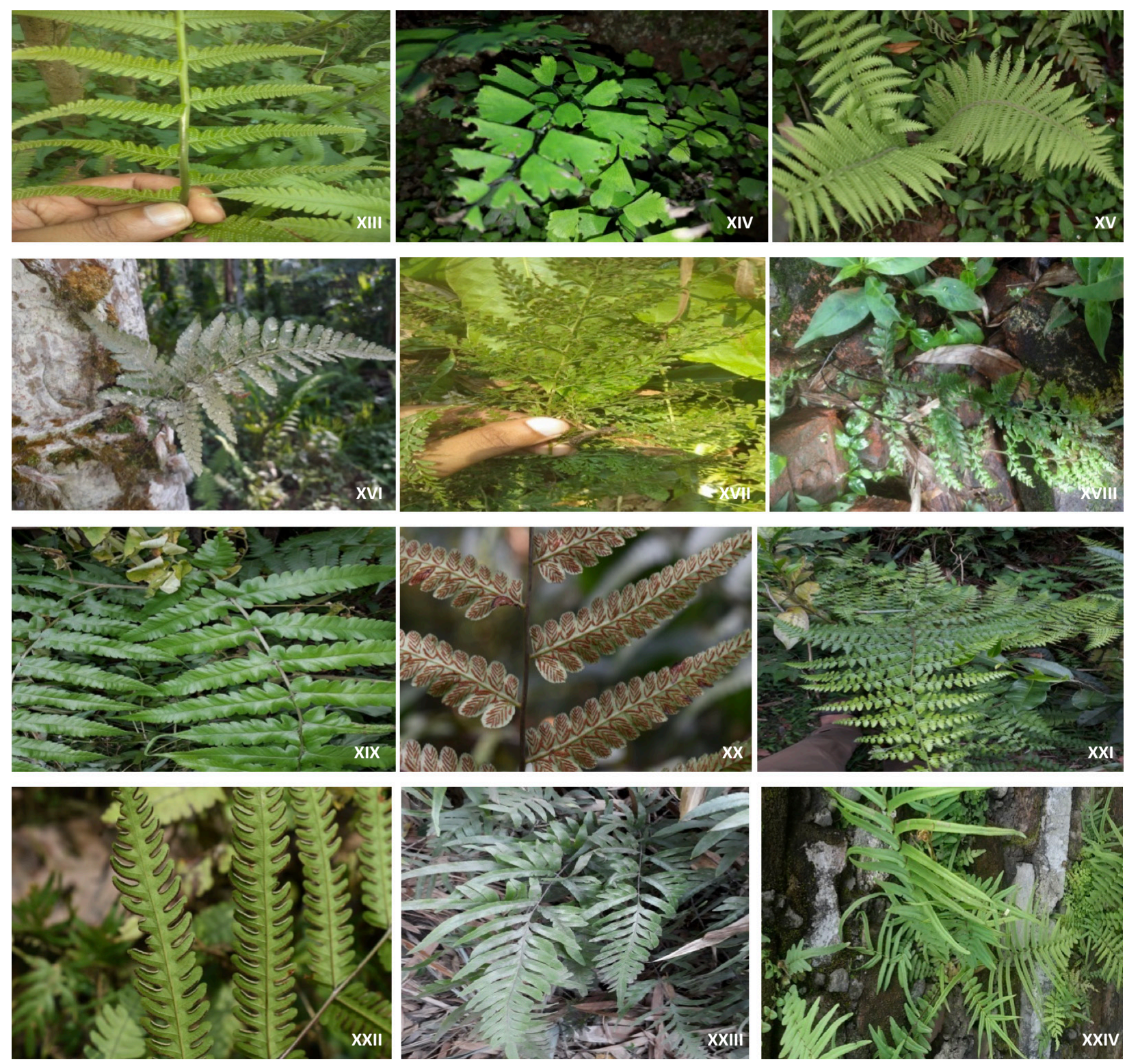

Image 1. XIII-Christella parasitica (L.) H.Lev. | XIV-Adiantum capillus-veneris L. | XV-Amblovenatum opulentumI (Kaulf.) J.P.Roux. | XVI-Araiostegia divaricata (BI.) M.Kato. | XVII-Sphenomeris chinensis (L.) Maxon | XVIII-Pityrogramma calomelanos (L.) Link. | XIXDiplazium esculentum (Retz.) Sw. | XX-Diplazium dilatatum BI. | XXI-Microlepia speluncae (L.) Moore | XXII-Pteris biaurita L. | XXIIIPteris semipinnata L. | XIV-Pteris vittata L. @ Pranjal Borah

and veins covered by short acicular or glandular hairs; veins up to 10 pairs, upper surface covered with thick, acicular hairs. Sori medial or submarginal on the veins up to five pairs.

26. Cyclosorus interruptus (Willd.) H. Ito.; Collection no. 5065.

Terestrial. Rhizome long creeping, clothed with scales at the apex; scales ovate, acuminate at apex, margin entire. Stipes slender, sparsely scaly and black at the base, glabrous and brown above, adaxially grooved, abaxially flattened. Lamina elliptic-lanceolate, simple pinnate with an apical pinnae; lateral pinnae numerous, sessile or very shortly stalked, basal pinnae not reduced, rachis grooved and hairy; veins slightly distinct below and densely covered by long, soft acicular hairs, upper surface glabrous; lamina pale green; sori medial on the veins, in two rows, arranged in V-shaped; indusia reniform, hairy. Spores pale brown.

\section{Aspleniaceae}

27. Asplenium nidus L.; Collection No. DRC- 5076

Epiphyte. Rhizome erect, short, stout, apex clothed 
with scales. Stipes dark to pale brown, glabrous above, scaly at base; lamina simple, lanceolate, gradually narrowed at both ends, glabrous; midrib strongly raised on the upper surface, veins nearly simple or 2-forked; almost parallel. Sori linear, borne along each veinlet on upper half of the lamina, nearly reaching margin from the midrib; indusia linear, narrow, superficially attached at base, slightly curved, greenish-grey. Spores light brown.

\section{Athyriaceae}

\section{Diplazium dilatatum Bl.}

Terrestrial. Rhizome erect, stout, apex densely clothed with scales; apex acuminate, margin with many teeth, thin, dark brown. Stipes scaly at base, glabrous above, abaxially rounded, adaxially grooved. Lamina ovate, bipinnate or tripinnatifid; primary pinnae seven pairs, alternate, shortly stalked or sessile, slightly ascending, pinnae up to 12 pairs, subopposite to alternate shortly stalked, simple or forked once, reaching the margin, texture herbaceous; lamina dark green above, pale green below, glabrous. Sori linear, confluent, indusia linear, entire, pale brown; sporangia slender stalked. Spores oval, pale brown.

\section{Diplazium esculentum (Retz.) Sw.}

Terrestrial. Rhizome erect, apex densely covered by scales, linear, lanceolate, apex long acuminate, darkbrown. Stipes stout, erect, sparsely scaly at base, darkbrown at base, pale-brown above. Lamina bipinnate at base, simple pinnate at apex, rarely simply pinnate, deltoid, apex acuminate, base truncate, basal pair of pinnae slightly reduced; pinnae up to seven pairs, basal one or two opposite or sub-opposite, others alternate; petiolate, narrowly deltoid, with a deeply lobed terminal pinnae, apex acuminate, lamina pale-green, hairs densely distributed all over the lamina. Sori in two rows near the margin, dark brown. Spores reniform, palebrown.

\section{Dryopteridaceae}

30. Bolbitis heteroclita (Presl.) Ching; Collection No. DRC- 5082.

Terrestrial. Rhizome long creeping, soft, brittle, apex clothed with ovate-lanceolate, darkbrown scales; Stipes green, sparsely covered by scales. Fronds usually in two or three alternate rows; they vary from simple to pinnate and loosely placed; pinnae usually trifoliate, simple leaves and terminal pinnae of pinnate leaves similar, veins prominent, lateral veins raised, secondary veins anatomising without included veinlets, marginal veins free. Fertile lamina simple or pinnate; apical pinnae lateral, fertile lanceolate with acute apex, margin smooth and shortly stalked. Sori covering the whole lower surface and brown.

\section{Davalliaceae}

31. Araiostegia divaricata (BI.) M.Kato.; Collection No. DRC- 5085.

Epiphyte. Rhizome creeping, densely scaly all over; scales, apex long acuminate, base broad, thin, transparent, brown. Stipes firm, erect, scaly at base, glabrous above, chestnut brown, tripinnatifid, deltoidlanceolate, apex acute or acuminate; apex acuminate, base cuneate; secondary pinnae up to 12 pairs, alternate, sessile or shortly stalked, margin deeply cut down to lobe nearly to the costules; margin sharply toothed or crenate; veins not conspicuous, uniform, free, not reaching the margin; lamina dark reddish-brown when dry, glabrous. Sori half cup-shaped, obliquely placed as regards the central veins in the tooth, submarginal, brownish; indusia tubular or half cup-shaped, as long as broad.

\section{Blechnaceae}

32. Blechnum orientale L.; Collection No. DRC- 5087.

Terrestrial. Rhizome creel, densely scaly, massive, linear-lanceolate, apex acuminate, shining, dark brown. Stipes tufted, erect, scaly at base, glabrous above, reddish-brown at the base, grey brown above. Lamina ovate to linear-lanceolate, apex acute, simple pinnate; costa grooved above, rounded below; veins slightly distinct, simple or forked once or two times, free; lamina pale green, glabrous above and below, glossy. Sori linear along either side of the costa, continuous nearly to the apex, dark brown; indusia narrow, firm with entire margin. Spores round to oval, translucent, yellowishbrown.

33. Stenochlaena palustris (Burm.f.) Bedd.; Collection No. DRC- 5090.

Climber. Rhizome scandant, long creeping, thick, sparsely scaly, often climbing on trees; scales ovate, apex acuminate, margin ciliated, dark-brown at the centre, pale-brown at the periphery. Lamina dimorphic; stramineous, glabrous, adaxially grooved, abaxially rounded; simple pinnate; pinnae 8-15 pairs, lanceolate, apex acuminate, hard, rachis similar to stipe; veins distinct, simple or rarely once forked, reaching the margin; lamina green, glabrous on both surfaces, shining. Fertile lamina borne at the distal part of the plant, more or less same size and shape with the sterile one, but pinnae much contracted. Sori densely covering the lower surface except midrib and the extreme apex; 
sporangia large, stalked, crowded. Spores monoiete, pale-green, spinulose.

\section{DISCUSSION}

The vegetation of the study area is mostly dominated by the angiosperms. Out of the 33 species of pteridophytes, two species belong to fern-allies and 31 species belong to true ferns. The two species of fernallies belong to the family Selaginellaceae. Considering the habitat, 25 plants are terrestrial, seven plants epiphytic, and one aquatic (Figure 2). Among them, three species are trees, three species are climbers and remaining 27 species are either herbs or shrubs (Figure 3). Three species namely Angiopteris evecta (G.Forst.) Hoffm., Cyathea spinulosa Wall. ex Hook. and Cyathea gigantea (Wall. ex Hook.) Holtt. are tree ferns. Microsorum pteropus (BI.) Copel. is the only aquatic fern found there. Lygodium flexuosum (L.) Sw., Lygodium japonicum (Thunb.) Sw., and Stenochlaena palustris (Burm. f.) Bedd. are climbers. Cyclosorus interruptus (Willd.) H. Ito is grown abundantly in swamp areas. Pityrogramma calomelanos (L.) Link. is commonly called

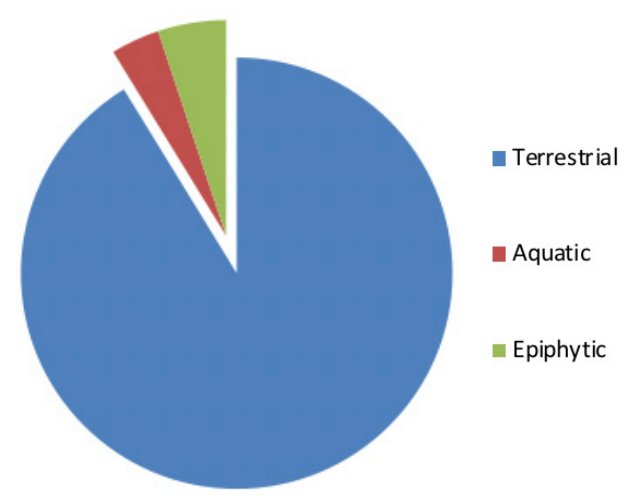

Figure 2. Habitats of pteridophytes.

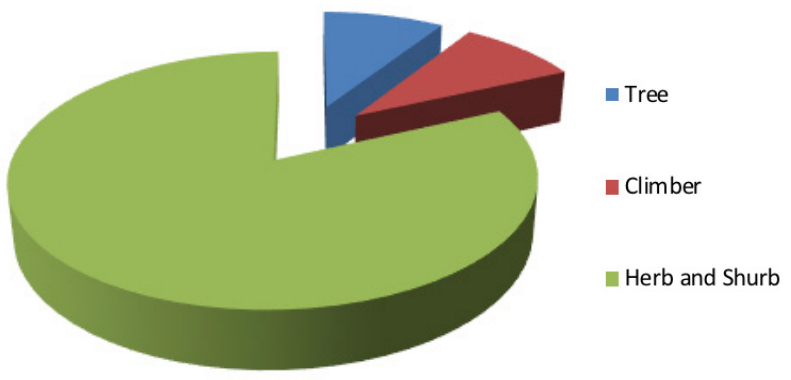

Figure 3. Different habits of pteridophytes.
Table 1. Number of pteridophyte taxa in genera and families.

\begin{tabular}{|c|c|c|}
\hline Family & Genus & Number of taxa \\
\hline Selaginellaceae & Selaginella & 2 \\
\hline Marrattiaceae & Angiopteris & 1 \\
\hline Gleicheniaceae & Dicranopteris & 1 \\
\hline \multirow{2}{*}{ Polypodiaceae } & Microsorum & 2 \\
\hline & Pyrrosia & 2 \\
\hline Lygodiaceae & Lygodium & 2 \\
\hline \multirow{5}{*}{ Pteridaceae } & Acrostichum & 1 \\
\hline & Pteris & 5 \\
\hline & Adiantum & 1 \\
\hline & Pityrogramma & 1 \\
\hline & Vittaria & 1 \\
\hline Cyatheaceae & Cyathea & 2 \\
\hline Dennstaedtiaceae & Microlepia & 1 \\
\hline Lindsaeaceae & Sphenomeris & 1 \\
\hline \multirow{3}{*}{ Thelypteridaceae } & Amblovenatum & 1 \\
\hline & Christella & 1 \\
\hline & Cyclosorus & 1 \\
\hline Aspleniaceae & Asplenium & 1 \\
\hline Athyriaceae & Diplazium & 2 \\
\hline Dryopteridaceae & Bolbitis & 1 \\
\hline Davalliaceae & Araiostegia & 1 \\
\hline \multirow{2}{*}{ Blechnaceae } & Blechnum & 1 \\
\hline & Stenochlaena & 1 \\
\hline
\end{tabular}

as silver fern due to its silver colour spores and found to be growing in the forest and also grow on stored bricks or nearby brick making industries. Epiphytic ferns Microsorum punctatum (L.) Copel. and Asplenium nidus L. are seen to be growing plentifully in tree trunks not only in the forest but also adjoining areas. Diplazium esculentum (Retz.) Sw. is widely used as vegetables in the nearby area. The fern species like Cyathea gigantea (Wall ex Hook.) Holtt. and Diplazium esculantum (Retz.) Sw. were observed to be taken as food by Trachypithecus geei during their study in Kakoijana reserve forest, Assam. However, no record traced the existence of this endangered primate species in the present study area. Due to the subsistence of other primate species including the endangered Hoolock Gibbons, these two fern species were kept unscathed and only digital photograph were taken.

The family Pteridaceae has the greatest number of genera, i.e. 5; among them, the genus Pteris has highest number of species. In India, the family Thelypteridaceae is represented by 80 species and 16 hybrids (Fraser- 
Jenkins et al. 2017) while only three species are recorded from the present study area. Most of the families and genera have a single number of species (Table 1 ).

\section{REFERENCES}

Baishya, A.K. \& R.R. Rao (1982). Ferns and Fern-allies of Meghalaya State, India. Scientific Publisher, Jodhpur, $162 \mathrm{pp}$

Borthakur, S.K., P. Deka \& K. Nath (2001). Illustrated Mannual of Ferns of Assam. Bishen Singh Mahendra Pal Singh, Dehra Dun, 468 pp.

Borthakur, S.K., D. Baro, A. Bawri \& A. Baro (2018). Flora of BTAD (Bodoland Territorial Area Districts, Assam), Pteridophytes and Gymnosperms. Eastern Book House Publisher, India, Vol.1, 152 pp.

Chandra, S. (2000). The Ferns of India (Enumeration, Synonyms \& Distribution). International Book Distributors, Dehradun, India.

Chapman, A.D. (2009). Numbers of Living Species in Australia and the World Report for the Australian Biological Resources Study Canberra, Australia, September 2009. http://wwwenvi ronmentgovau/ biodiversity/abrs/publications/other/species/numbers/indexhtml.

Devi, J. \& H.C. Majumdar (2003). A study on Pteridophytic flora Byrnihat and Umtru area of Meghalaya State. Plant Archives 3(2): 207-213.

Dixit, R.D. (1984). A Census of the Indian Pteridophytes. Botanical Survey of India, Howrah.

Dutta, A.K., T.K. Dutta \& K.K. Gupta (1980). A tentative accounting of the forest flora of North Cachar Hills and Barail range-II: Enumeration of spores of Pteridophytes. Indian Forester 106: 34-40.

Fraser-Jenkins, C.R. (2009). A brief comparison of modern pteridophyte classifications (families and genera in India). Indian Fern Journal 26 107-126.

Fraser-Jenkins, C.R., K.N. Gandhi, B.S. Kholia \& A. Benniamin (2017) An annonated checklist of Indian pteridophytes part-1. Bishen Singh Mahendra Pal Singh, Dehradun, India, 562 pp.
Handique, P.J. \& G. Konger (1986). A list of Ferns and Fern allies of greater Guwahati (Assam) with their ecological adaptation. Mendel 3(2): 130-135.

Jain, S.K. \& R.R. Rao (1977). A Handbook of Field and Herbarium Methods. Today \& Tomorrow's Printers and Publishers, New Delhi, 157 pp.

Jamir, N.S. \& R.R. Rao (1988). The Ferns of Nagaland. Bishen Singh Mahendra Pal Singh, Dehra Dun, India, 426 pp.

Kachroo, P. (1953). Ferns of Assam. Journal of Asiatic Society, Bengal. 29: 161-174.

Kachroo, P., S.S. Bir \& S.M. Vasudeva (1989). Pteridophytic flora of North-Eastern India-II (families: CryptogramaceaeThelypteridaceae. Indian Fern Journal 6: 78-99.

Kalita, P.C. (2015). Survey for pteridophytic diversity in Madan Kamdev Hill region of Assam, India. Pleione 9(2): 376-400.

Moran, R.C. (2015). An overview of ferns and lycophytes Opening Symposium Abstract (p5) In 'Next Generation Pteridology' [An International Conference on Lycophyte and Fern Research], Smithsonian Institution and US Botanic Garden, Washington DC; June 1-5, 2015 Book of Program and Abstracts, Pp 74

Panigrahi, G. (1960). Pteridophytes of eastern India-1 Enumeration of the species collected and their nomenclature. Bulletin of Botanical Survey of India 2: 309-314.

Panigrahi, G. (1968). Studies in the Polypodiaceae in eastern India: distribution and ecology. Journal of Indian Botanical Society 47: 1-6.

Panigrahi, G. \& S. Choudhury (1961). Taxonomic studies on the Aspidiaceae of Eastern India. Proceeding of Indian Science Congress 48: 272.

Panigrahi, G. \& S. Choudhury (1962). Enumeration and distribution of fern allies in Eastern India. Proceeding of Indian Science Congress 49: 255-256.

Sen, A. \& P.D. Ghosh (2011). A note of the ethnobotanical studies of some pteridophytes in Assam. Indian Journal of Traditional Knowledge 10(2): 292-295. 
Dr. Albert G. Orr, Griffith University, Nathan, Australia

Dr. Sameer Padhye, Katholieke Universiteit Leuven, Belgium

Dr. Nancy van der Poorten, Toronto, Canada

Dr. Kareen Schnabel, NIWA, Wellington, New Zealand

Dr. R.M. Sharma, (Retd.) Scientist, Zoological Survey of India, Pune, India

Dr. Manju Siliwal, WILD, Coimbatore, Tamil Nadu, India

Dr. G.P. Sinha, Botanical Survey of India, Allahabad, India

Dr. K.A. Subramanian, Zoological Survey of India, New Alipore, Kolkata, India

Dr. P.M. Sureshan, Zoological Survey of India, Kozhikode, Kerala, India

Dr. R. Varatharajan, Manipur University, Imphal, Manipur, India

Dr. Eduard Vives, Museu de Ciències Naturals de Barcelona, Terrassa, Spain

Dr. James Young, Hong Kong Lepidopterists' Society, Hong Kong

Dr. R. Sundararaj, Institute of Wood Science \& Technology, Bengaluru, India

Dr. M. Nithyanandan, Environmental Department, La Ala Al Kuwait Real Estate. Co. K.S.C., Kuwait

Dr. Himender Bharti, Punjabi University, Punjab, India

Mr. Purnendu Roy, London, UK

Dr. Saito Motoki, The Butterfly Society of Japan, Tokyo, Japan

Dr. Sanjay Sondhi, TITLI TRUST, Kalpavriksh, Dehradun, India

Dr. Nguyen Thi Phuong Lien, Vietnam Academy of Science and Technology, Hanoi, Vietnam

Dr. Nitin Kulkarni, Tropical Research Institute, Jabalpur, India

Dr. Robin Wen Jiang Ngiam, National Parks Board, Singapore

Dr. Lional Monod, Natural History Museum of Geneva, Genève, Switzerland.

Dr. Asheesh Shivam, Nehru Gram Bharti University, Allahabad, India

Dr. Rosana Moreira da Rocha, Universidade Federal do Paraná, Curitiba, Brasil

Dr. Kurt R. Arnold, North Dakota State University, Saxony, Germany

Dr. James M. Carpenter, American Museum of Natural History, New York, USA

Dr. David M. Claborn, Missouri State University, Springfield, USA

Dr. Kareen Schnabel, Marine Biologist, Wellington, New Zealand

Dr. Amazonas Chagas Júnior, Universidade Federal de Mato Grosso, Cuiabá, Brasil

Mr. Monsoon Jyoti Gogoi, Assam University, Silchar, Assam, India

Dr. Heo Chong Chin, Universiti Teknologi MARA (UiTM), Selangor, Malaysia

Dr. R.J. Shiel, University of Adelaide, SA 5005, Australia

Dr. Siddharth Kulkarni, The George Washington University, Washington, USA

Dr. Priyadarsanan Dharma Rajan, ATREE, Bengaluru, India

Dr. Phil Alderslade, CSIRO Marine And Atmospheric Research, Hobart, Australia

Dr. John E.N. Veron, Coral Reef Research, Townsville, Australia

Dr. Daniel Whitmore, State Museum of Natural History Stuttgart, Rosenstein, Germany.

Dr. Yu-Feng Hsu, National Taiwan Normal University, Taipei City, Taiwan

Dr. Keith V. Wolfe, Antioch, California, USA

Dr. Siddharth Kulkarni, The Hormiga Lab, The George Washington University, Washington,

D.C., USA

Dr. Tomas Ditrich, Faculty of Education, University of South Bohemia in Ceske

Budejovice, Czech Republic

Dr. Mihaly Foldvari, Natural History Museum, University of Oslo, Norway

Dr. V.P. Uniyal, Wildlife Institute of India, Dehradun, Uttarakhand 248001, India

Dr. John T.D. Caleb, Zoological Survey of India, Kolkata, West Bengal, India

Dr. Priyadarsanan Dharma Rajan, Ashoka Trust for Research in Ecology and the Environment (ATREE), Royal Enclave, Bangalore, Karnataka, India

\section{Fishes}

Dr. Neelesh Dahanukar, IISER, Pune, Maharashtra, India

Dr. Topiltzin Contreras MacBeath, Universidad Autónoma del estado de Morelos, México

Dr. Heok Hee Ng, National University of Singapore, Science Drive, Singapore

Dr. Rajeev Raghavan, St. Albert's College, Kochi, Kerala, India

Dr. Robert D. Sluka, Chiltern Gateway Project, A Rocha UK, Southall, Middlesex, UK

Dr. E. Vivekanandan, Central Marine Fisheries Research Institute, Chennai, India

Dr. Davor Zanella, University of Zagreb, Zagreb, Croatia

Dr. A. Biju Kumar, University of Kerala, Thiruvananthapuram, Kerala, India

Dr. Akhilesh K.V., ICAR-Central Marine Fisheries Research Institute, Mumbai Research

Centre, Mumbai, Maharashtra, India

Dr. J.A. Johnson, Wildlife Institute of India, Dehradun, Uttarakhand, India

\section{Amphibians}

Dr. Sushil K. Dutta, Indian Institute of Science, Bengaluru, Karnataka, India

Dr. Annemarie Ohler, Muséum national d'Histoire naturelle, Paris, France

\section{Reptiles}

Dr. Gernot Vogel, Heidelberg, Germany

Dr. Raju Vyas, Vadodara, Gujarat, India

Dr. Pritpal S. Soorae, Environment Agency, Abu Dubai, UAE.

Prof. Dr. Wayne J. Fuller, Near East University, Mersin, Turkey

Prof. Chandrashekher U. Rivonker, Goa University, Taleigao Plateau, Goa. India

Dr. S.R. Ganesh, Chennai Snake Park, Chennai, Tamil Nadu, India

Dr. Himansu Sekhar Das, Terrestrial \& Marine Biodiversity, Abu Dhabi, UAE
Birds

Dr. Hem Sagar Baral, Charles Sturt University, NSW Australia

Dr. Chris Bowden, Royal Society for the Protection of Birds, Sandy, UK

Dr. Priya Davidar, Pondicherry University, Kalapet, Puducherry, India

Dr. J.W. Duckworth, IUCN SSC, Bath, UK

Dr. Rajah Jayapal, SACON, Coimbatore, Tamil Nadu, India

Dr. Rajiv S. Kalsi, M.L.N. College, Yamuna Nagar, Haryana, India

Dr. V. Santharam, Rishi Valley Education Centre, Chittoor Dt., Andhra Pradesh, India

Dr. S. Balachandran, Bombay Natural History Society, Mumbai, India

Mr. J. Praveen, Bengaluru, India

Dr. C. Srinivasulu, Osmania University, Hyderabad, India

Dr. K.S. Gopi Sundar, International Crane Foundation, Baraboo, USA

Dr. Gombobaatar Sundev, Professor of Ornithology, Ulaanbaatar, Mongolia

Prof. Reuven Yosef, International Birding \& Research Centre, Eilat, Israel

Dr. Taej Mundkur, Wetlands International, Wageningen, The Netherlands

Dr. Carol Inskipp, Bishop Auckland Co., Durham, UK

Dr. Tim Inskipp, Bishop Auckland Co, Durham, UK

Dr. V. Gokula, National College, Tiruchirappalli, Tamil Nadu, India

Dr. Arkady Lelej, Russian Academy of Sciences, Vladivostok, Russia

Dr. Simon Dowell, Science Director, Chester Zoo, UK

Dr. Mário Gabriel Santiago dos Santos, Universidade de Trás-os-Montes e Alto Douro,

Quinta de Prados, Vila Real, Portugal

Dr. Grant Connette, Smithsonian Institution, Royal, VA, USA

Dr. M. Zafar-ul Islam, Prince Saud Al Faisal Wildlife Research Center, Taif, Saudi Arabia

Mammals

Dr. Giovanni Amori, CNR - Institute of Ecosystem Studies, Rome, Italy

Dr. Anwaruddin Chowdhury, Guwahati, India

Dr. David Mallon, Zoological Society of London, UK

Dr. Shomita Mukherjee, SACON, Coimbatore, Tamil Nadu, India

Dr. Angie Appel, Wild Cat Network, Germany

Dr. P.O. Nameer, Kerala Agricultural University, Thrissur, Kerala, India

Dr. Ian Redmond, UNEP Convention on Migratory Species, Lansdown, UK

Dr. Heidi S. Riddle, Riddle's Elephant and Wildlife Sanctuary, Arkansas, USA

Dr. Karin Schwartz, George Mason University, Fairfax, Virginia.

Dr. Lala A.K. Singh, Bhubaneswar, Orissa, India

Dr. Mewa Singh, Mysore University, Mysore, India

Dr. Paul Racey, University of Exeter, Devon, UK

Dr. Honnavalli N. Kumara, SACON, Anaikatty P.O., Coimbatore, Tamil Nadu, India

Dr. Nishith Dharaiya, HNG University, Patan, Gujarat, India

Dr. Spartaco Gippoliti, Socio Onorario Società Italiana per la Storia della Fauna "Giuseppe

Altobello", Rome, Italy

Dr. Justus Joshua, Green Future Foundation, Tiruchirapalli, Tamil Nadu, India

Dr. H. Raghuram, The American College, Madurai, Tamil Nadu, India

Dr. Paul Bates, Harison Institute, Kent, UK

Dr. Jim Sanderson, Small Wild Cat Conservation Foundation, Hartford, USA

Dr. Dan Challender, University of Kent, Canterbury, UK

Dr. David Mallon, Manchester Metropolitan University, Derbyshire, UK

Dr. Brian L. Cypher, California State University-Stanislaus, Bakersfield, CA

Dr. S.S. Talmale, Zoological Survey of India, Pune, Maharashtra, India

Prof. Karan Bahadur Shah, Budhanilakantha Municipality, Kathmandu, Nepal

Dr. Susan Cheyne, Borneo Nature Foundation International, Palangkaraja, Indonesia

Dr. Hemanta Kafley, Wildlife Sciences, Tarleton State University, Texas, USA

\section{Other Disciplines}

Dr. Aniruddha Belsare, Columbia MO 65203, USA (Veterinary)

Dr. Mandar S. Paingankar, University of Pune, Pune, Maharashtra, India (Molecular)

Dr. Jack Tordoff, Critical Ecosystem Partnership Fund, Arlington, USA (Communities)

Dr. Ulrike Streicher, University of Oregon, Eugene, USA (Veterinary)

Dr. Hari Balasubramanian, EcoAdvisors, Nova Scotia, Canada (Communities)

Dr. Rayanna Hellem Santos Bezerra, Universidade Federal de Sergipe, São Cristóvão, Brazil

Dr. Jamie R. Wood, Landcare Research, Canterbury, New Zealand

Dr. Wendy Collinson-Jonker, Endangered Wildlife Trust, Gauteng, South Africa

Dr. Rajeshkumar G. Jani, Anand Agricultural University, Anand, Gujarat, India

Dr. O.N. Tiwari, Senior Scientist, ICAR-Indian Agricultural Research Institute (IARI), New

Delhi, India

Dr. L.D. Singla, Guru Angad Dev Veterinary and Animal Sciences University, Ludhiana, India

Dr. Rupika S. Rajakaruna, University of Peradeniya, Peradeniya, Sri Lanka

Dr. Bahar Baviskar, Wild-CER, Nagpur, Maharashtra 440013, India

Reviewers 2018-2020

Due to pausity of space, the list of reviewers for 2018-2020 is available online.
The opinions expressed by the authors do not reflect the views of the Journal of Threatened Taxa, Wildlife Information Liaison Development Society, Zoo Outreach Organization, or any of the partners. The journal, the publisher, the host, and the partners are not responsible for the accuracy of the political boundaries shown in the maps by the authors.

\footnotetext{
Print copies of the Journal are available at cost. Write to:

The Managing Editor, JoTT,

c/o Wildlife Information Liaison Development Society,

No. 12, Thiruvannamalai Nagar, Saravanampatti - Kalapatti Road,

Saravanampatti, Coimbatore, Tamil Nadu 641035, India

ravi@threatenedtaxa.org
} 


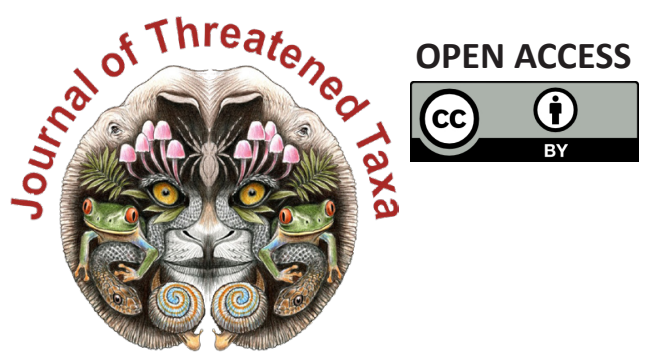

www.threatenedtaxa.org

The Journal of Threatened Taxa (JoTT) is dedicated to building evidence for conservation globally by publishing peer-reviewed articles online every month at a reasonably rapid rate at www.threatenedtaxa.org. All articles published in JoTT are registered under Creative Commons Attribution 4.0 International License unless otherwise mentioned. JoTT allows allows unrestricted use, reproduction, and distribution of articles in any medium by providing adequate credit to the author(s) and the source of publication.

\section{ISSN $0974-7907$ (Online) | ISSN $0974-7893$ (Print)}

\section{October 2021 | Vol. 13 | No. 12 | Pages: 19675-19886 \\ Date of Publication: 26 October 2021 (Online \& Print) DOI: 10.11609/jott.2021.13.12.19675-19886}

Articles

Roosting habits and habitats of the Indian Flying Fox Pteropus medius Temminck, 1825 in the northern districts of Tamil Nadu, India

- M. Pandian \& S. Suresh, Pp. 19675-19688

Diversity and distribution of avifauna at Warathenna-Hakkinda Environmental Protection Area in Kandy, Sri Lanka

- Dinelka Thilakarathne, Tithira Lakkana, Gayan Hirimuthugoda, Chaminda Wijesundara \& Shalika Kumburegama, Pp. 19689-19701

Grass species composition in tropical forest of southern India

- M. Ashokkumar, S. Swaminathan \& R. Nagarajan, Pp. 19702-19713

\section{Communications}

Habitat use and conservation threats to Wild Water Buffalo Bubalus arnee (Mammalia: Artiodactyla: Bovidae) in Koshi Tappu Wildlife Reserve, Nepal

- Reeta Khulal, Bijaya Neupane, Bijaya Dhami, Siddhartha Regmi, Ganesh Prasad Tiwari \& Manita Parajuli, Pp. 19714-19724

Get my head around owls: people perception and knowledge about owls of Andaman Islands

- Shanmugavel Sureshmarimuthu, Santhanakrishnan Babu, Nagaraj Rajeshkumar \& Honnavalli Nagaraj Kumara, Pp. 19725-19732

Abundance and diversity of threatened birds in Nangal Wetland, Punjab, India - Rajwinder Kaur \& Onkar Singh Brraich, Pp. 19733-19742

Evaluation of fish diversity and abundance in the Kabul River with comparisons between reaches above and below Kabul City, Afghanistan

- Ugyen Kelzang, Ahmad Farid Habibi \& Ryan J. Thoni, Pp. 19743-19752

New record of Myrmarachne melanocephala MacLeay, 1839 (Araneae: Salticidae) from Jharkhand, India and biogeographical implications of the co-occurrence of its ant model Tetraponera rufonigra Jerdon, 1851

- Rahul Kumar, Mirtunjay Sharma \& Ajay Kumar Sharma, Pp. 19753-19761

Diversity of spiders (Arachnida: Araneae) and the impact of pruning in Indian sandalwood plantations from Karnataka, India

-S. Padma 1 \& R. Sundararaj, Pp. 19762-19772

New records of cheilostome Bryozoa from the eastern coast of India encrusting on the exoskeleton of live horseshoe crabs of Indian Sundarbans

- Swati Das, Maria Susan Sanjay, Basudev Tripathy, C. Venkatraman \& K.A. Subramanian, Pp. 19773-19780

On the pteridophytes of Bherjan-Borajan-Padumoni Wildlife Sanctuary, Assam, India - Pranjal Borah \& Jayanta Barukial, Pp. 19781-19790

Population status of Heritiera fomes Buch.-Ham., a threatened species from Mahanadi Mangrove Wetland, India

- Sudam Charan Sahu, Manas Ranjan Mohanta \& N.H. Ravindranath, Pp. 19791-19798

Additions to the lichenized and lichenicolous fungi of Jammu \& Kashmir from Kishtwar High Altitude National Park

- Vishal Kumar, Yash Pal Sharma, Siljo Joseph, Roshinikumar Ngangom \& Sanjeeva Nayaka, Pp. 19799-19807

\section{Short Communications}

Is release of rehabilitated wildlife with embedded lead ammunition advisable? Plumbism in a Jaguar Panthera Onca (Mammalia: Carnivora: Felidae), survivor of gunshot wounds - Eduardo A. Díaz, Carolina Sáenz, E. Santiago Jiménez, David A. Egas \& Kelly Swing, Pp. 19808-19812

New record of the Sewing Needle Zipper Loach Paracanthocobitis linypha Singer \& Page, 2015 (Teleostei: Cypriniformes: Nemacheilidae) from the Chindwin drainage of Manipur, India

- Yumnam Rameshori, Yengkhom Chinglemba \& Waikhom Vishwanath, Pp. 19813-19817

Field identification characters to diagnose Microhyla mukhlesuri from closely related M. mymensinghensis (Amphibia: Microhylidae) and range extension of $M$. mukhlesuri up to West Bengal State, India

- Suman Pratihar \& Kaushik Deuti, Pp. 19818-19823
First report of Scipinia horrida (Stål) (Heteroptera: Reduviidae) from Assam, with comments on related genus Irantha Stål

- Anjana Singha Naorem, Santana Saikia, Anandita Buragohain, Rubina Azmeera Begum, Swapnil S. Boyane \& Hemant V. Ghate, Pp. 19824-19830

Flesh fly (Diptera: Sarcophagidae): male terminalia, diversity and expanded geographical distribution from India

- Kanholi Sreejith, Shuvra Kanti Sinha, Santanu Mahato \& Edamana Pushpalatha, Pp. 1983119836

Checklist of moths (Heterocera) of Tadong, Sikkim, India

- Prayash Chettri, Yuki Matsui, Hideshi Naka \& Archana Tiwari, Pp. 19837-19848

New distribution records of Begonia L., B. murina Craib and B. poilanei Kiew (Begoniaceae: Cucurbitales) for Laos

- Phongphayboun Phonepaseuth, Phetlasy Souladeth, Soulivanh Lanorsavanh, Shuichiro Tagane, Thyraphon Vongthavone \& Keooudone Souvannakhoummane Pp. 19849-19854

Notes

A recent sighting of the Stripe-backed Weasel Mustela strigidorsa (Mammalia: Carnivora: Mustelidae) in Hkakabo Razi Landscape, Myanmar

- Sai Sein Lin Oo, Tun Tun, Kyaw Myo Naing \& Paul Jeremy James Bates, Pp. 19855-19859

Are the uplifted reef beds in North Andaman letting nesting Olive Ridley Sea Turtle Lepidochelys olivacea stranded?

- Nehru Prabakaran, Anoop Raj Singh \& Vedagiri Thirumurugan, Pp. 19860-19863

First record of the orb-weaving spider Araneus tubabdominus Zhu \& Zhang, 1993 (Araneae: Araneidae) from India

- Souvik Sen, John T.D. Caleb \& Shelley Acharya, Pp. 19864-19866

The genus Catapiestus Perty, 1831 (Coleoptera: Tenebrionidae: Cnodalonini) from Arunachal Pradesh with one new record to India

- V.D. Hegde \& Sarita Yadav, Pp. 19867-19869

Rediscovery and extended distribution of Indigofera santapaui Sanjappa (Leguminosae: Papilionoideae) from the states of Maharashtra and Gujarat, India

- Kumar Vinod Chhotupuri Gosavi, Sanjay Gajanan Auti, Sharad Suresh Kambale \& Munivenkatappa Sanjappa, Pp. 19870-19873

Additional distribution records of Ceropegia anjanerica, an endemic and 'Endangered' lantern flower of the northern Western Ghats, India

- Samir Shrikant Maity, Ajay Natha Gangurde, Sharad Suresh Kambale, Avinash Ramchandra Gholave, Avinash Asraji Adsul, Ganesh Babaso Pawar \& Kumar Vinod Chhotupuri Gosavi, Pp. 19874-19877

Notes on the extended distribution of Impatiens megamalayana, a recently described balsam in Western Ghats, India

- Anoop P. Balan \& A.J. Robi, Pp. 19878-19883

Book Review

A look over on the scented tree of India (Santalum album - S. Suresh Ramanan \& A. Arunachalam, Pp. 19884-19886
Publisher \& Host
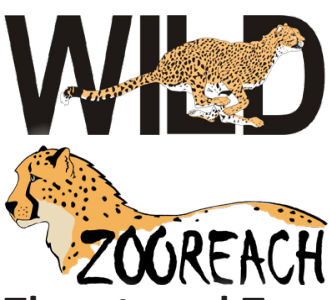

Threatened Taxa 